\title{
Improvement for sensitivity of biosensor with total internal reflection imaging ellipsometry (TIRIE)
}

\author{
Li Liu ${ }^{\text {a,b }}$, Yan-yan Chen ${ }^{\text {, }}$, Yong-hong Meng a , She Chen a , Gang Jin ${ }^{\text {a,* }}$ \\ a National Microgravity Laboratory, Institute of Mechanics, Chinese Academy of Sciences, \#15, Bei-si-huan West Rd., Beijing 100190, China \\ b Graduate School, Chinese Academy of Sciences, \#19, Yu-quan Rd., Beijing 100049, China \\ c Institute of Nano-tech and Nano-bionics, Chinese Academy of Sciences, Suzhou 215125, China
}

\section{A R T I C L E I N F O}

Available online 23 December 2010

\section{Keywords:}

Sensitivity improvement

Imaging ellipsometry

Total internal reflection

Biosensor

HbsAg detection

\begin{abstract}
A B S T R A C T
The biosensor based on the total internal reflection imaging ellipsometry (TIRIE) is realized as an automatic analysis method for protein interaction processes in real-time, with high throughput and label-free. An evanescent wave is used as the optical probe to monitor bio-molecular interactions on a chip surface with a high sensitivity due to its phase sensitive property. In this paper, the technique is optimized with a polarization setting, a spectroscopic light source and a low noise CCD detector to improve the performance of the biosensor in sensitivity and detection limit, as evidenced by a quantitative detection of Hepatitis B virus surface antigen (HbsAg) with concentrations of 8, 16, 32, 64, 125 and $250 \mathrm{ng} / \mathrm{ml}$. The sensitivity is increased by one order of magnitude and the detection limit has been extended more than 50 times for HbsAg detection. (C) 2011 Elsevier B.V. All rights reserved.
\end{abstract}

\section{Introduction}

In recent years, label-free optical biosensors are increasingly used in bio-chemical analysis, as well as in numerous applications of biomedical researches, such as those related to pharmaceuticals, clinic and environmental monitoring [1-3]. In biological and medical studies, optical biosensors of high sensitivity and high-throughput are highly desirable. Conventional surface plasmon resonance (SPR) biosensor is the most popular label-free optical biosensor, and has been widely used in biomolecule interaction analysis for years, since it is introduced [4-6] for the detection of biological and chemical reactions in thin films and monolayer [7]. However, some limitations in the SPR hamper its further applications. As a dip in angular or spectral interrogation of the reflected light intensity is used as the result of SPR [8], and the optical sampling of this reflected light intensity is not sensitive to the change on the interface, it does not usually make a very sensitive and accurate detection. In addition, the throughput of the biosensor is limited [8]. The BiaCore flexchip SPR biosensor is an advanced instrument for high-throughput detection, which can monitor 400 interactions of biomolecules simultaneously $[9,10]$, however the high throughput is achieved through a sacrifice in the sample consumption and the detection concentration range of the biosensor from the order of $10 \mu \mathrm{l}$ and $\mathrm{ng} / \mathrm{ml}$ to that of the $\mathrm{ml}$ and $100 \mu \mathrm{g} / \mathrm{ml}$, respectively. In order to improve the sensitivity of the conventional SPR biosensor, the ellipsometry method is applied.

\footnotetext{
* Corresponding author.

E-mail address: gajin@imech.ac.cn (G. Jin).
}

Ellipsometry is a sensitive optical technique for the detection of the thickness and refractive index of thin films at the interface, based on measurements of the change in the polarization state of a light beam reflected by a surface [11]. It is a phase-sensitive method and has high sensitivity which reaches to the order of sub-angstrom of a thin film at the interface. The total internal reflection ellipsometry (TIRE or surface plasmon enhanced ellipsometry) biosensor is developed from the conventional SPR biosensor, based on the ellipsometer operating in the total internal-reflection mode $[12,13]$. The polarization control of the ellipsometry in the SPR biosensor is applied for the biomolecule detection [7,14-16]. Its sensitivity is shown to be 10 times higher than that of the conventional SPR biosensor [13], however, highthroughput is a difficult part in design. Imaging approach is an effective method for high-throughput detection. The total internal reflection imaging ellipsometry (TIRIE) concerns a biosensor of imaging ellipsometry operating in an internal reflection mode. As described in [17], TIRIE biosensor is based on the phase sensitive ellipsometric property and the large view field property of the imaging ellipsometry. In addition, the micro-fluidic reactor array system is applied for biomolecule interactions and sampling, to provide the high sensitivity and high throughput property.

In our previous work [17], kinetic measurements of biomolecule interactions with high throughput were simultaneously obtained with the TIRIE biosensor system, but the sensitivity and the detection limit of the biosensor system were not quite satisfactory. In this study, the performance of the TIRIE biosensor is improved. Three optimization procedures are adopted. Firstly, the optical settings of polarization components are optimized for the maximum sensitivity. Secondly, with the consideration of the dispersion of materials used in the 
sensing surface of the biosensor, the spectroscopic light source is applied instead of single wavelength, which may provide an optimized dispersive choice. At last, a low noise CCD detector is adopted to decrease the thermal noise of the imaging system so that the sensitivity and the detection limit are much improved, as shown in a demonstration of $\mathrm{HbsAg}$ and its antibody interaction process.

\section{Principle}

The principle of applying the TIRIE biosensor to detect the kinetic process of the protein interaction is illustrated in Fig. 1. Generally, a ligand and its receptor such as an antibody and its corresponding antigen could be assembled into a bio-complex due to their affinity. The optical biosensor is formed in a sense that each reactant as a ligand is immobilized to a surface to form a monolayer as a bioprobe with bioactivity. The other reactant as the analyte (or receptor) is in a solution. The bioprobe is exposed to the solution with the analyte. The analyte would interact with its corresponding ligand and assembled into a complex due to their specific bio-affinity. The molecule mass surface concentration on the surface where the interaction takes place would become higher than before exposure to the analyte solution. An increase of the surface concentration would indicate that the solution contains some amount of the receptor against the ligand on the surface.

In the optical system, a coupling prism is used for the internal reflection. The incident angle of the TIRIE biosensor is fixed and it is larger than the total internal reflection angle. An evanescent wave appears at the interface of the substrate and the protein layer. The polarization of the evanescent wave is modified by depositions of biomolecule layers on the surface so that the polarization state of the reflection changes at the same time.

The micro-fluidic reactor array system coupled with an optical prism on the back side of the substrate is utilized for the biomolecule interaction and sampling. The PBS buffer was used as liquid background, the ligand layer and the complex layer on the substrate in individual cells can be visualized in the ellipsometric image with the reflection intensity expressed in grayscales. The variation of the protein surface concentration on the substrate is reflected by the grayscale values of the image. The surface concentration distribution of the protein layer can be easily seen in three parts with different grayscales. The whole kinetic process of the biomolecule interaction in one cell at each moment is captured with the optical imaging in grayscales. Finally, a kinetic expression reflected by a series of images in grayscales with time is used to show the process.

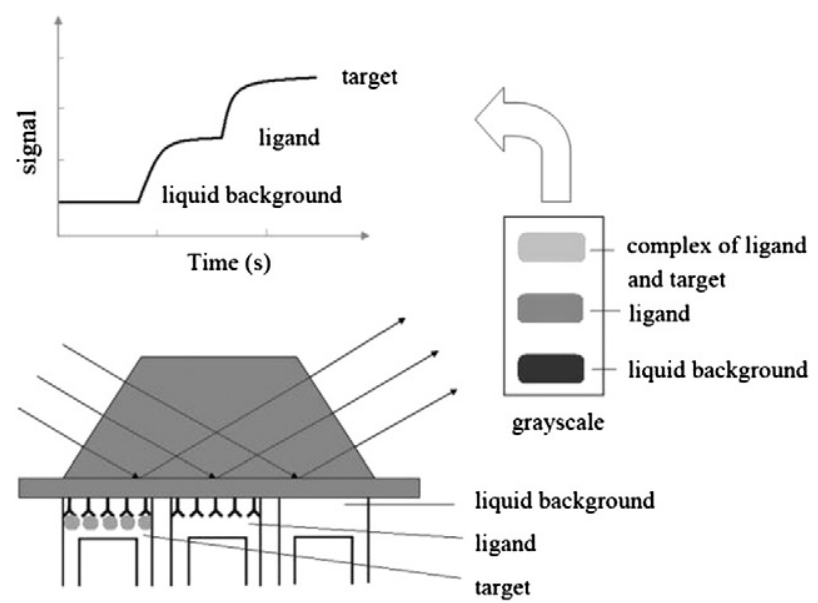

Fig. 1. Principle of the TIRIE biosensor, the biomolecule interaction on the substrate surface and its visualization with a series of images in grayscales with time.

\section{TIRIE biosensor setup}

A schematic diagram of the TIRIE biosensor setup is shown in Fig. 2. The TIRIE biosensor is a coupling of the optical system and a microfluidic protein reactor array system. The optical system uses an imaging ellipsometry with a light source of Xe lamp and a wavelength selector to form an expanded collimating light probe of a single wavelength. The light beam from the optical fiber goes through the polarizer and the compensator (a quarter wave plate) and penetrates into the coupling prism perpendicularly. The light beam is reflected totally at the interface of the substrate and passes through an analyzer, an imaging lens to the imaging device of CCD. The azimuth setting of the polarizer, the compensator and the analyzer is adjusted in a range from $0^{\circ}$ to $180^{\circ}$.

The micro-fluidic protein reactor array system is made of (polydimethylsiloxane) (PDMS), which includes a $3 \times 8$ cell array and each cell volume is about $150 \mathrm{nl}$. The micro-array reactor is attached to a gold layer surface on glass to form 24 individual cell arrays. Each cell has an inlet and an outlet for the solution. Any kinds of molecule solution can be individually delivered to each cell by the micro-fluidic system. By the micro-fluidic reactor array system, the sample consumption of the biomolecule solution reaches about $15 \mu \mathrm{l}$ and the test time normally is effectively minimized to tens of minutes. The optimized flux is $5 \mu \mathrm{l} / \mathrm{min}$. Moreover, a high throughput ( 24 channels) detection can be easily achieved with the combined TIRIE with a micro-fluidic reactor array system.

\section{Optimization of sensitivity with optical settings}

The sensitivity is an important parameter related to the biosensor performance. It is defined as the magnitude of the biosensor transduction signal change in response to the change in the analyte [18]. For the TIRIE biosensor, the intensity contrast $D=\frac{\Delta I}{I_{i}}$ is taken as the transduction signal. Here, $\Delta I$ is the intensity difference between biomolecule layers with different concentrations and the substrate and $I_{i}$ is defined as the input intensity. The sensitivity of the TIRIE biosensor is the ratio of the differential signal over the differential concentration of target biomolecule. In another word, if the intensity contrast is measured against changes in concentrations, the sensitivity is the slope of the generated curve [19]. Apart from the sensitivity, the detection limit is also an important index for the performance of the biosensor, which is defined as the lowest concentration that the biosensor can accurately detect.

The effects of the azimuth setting of the polarized elements and the choice of the wavelength on the sensitivity are first considered. At the same time, the imaging device with low noise is adopted to improve the imaging quality and the ratio of the signal and the noise.

\subsection{Polarization}

Polarization plays an important role in the biosensor system when the angle of incidence, the setting of the wavelength and the optical parameters of the sample are fixed. In order to illustrate the function of the polarization, the theory of the conventional polarization setting with polarized elements of polarizer $P$, compensator $C$, sample $S$ and an analyzer $A$ for the imaging (PCSA) is used. The intensity of the polarized imaging system is expressed as:

$$
\begin{aligned}
I= & K\left(\left|R_{S}\right|^{2} / 2\right)\left\{[1+\cos 2 C \cos 2(P-C)] \cos ^{2} A \tan ^{2} \psi\right. \\
& +[1-\cos 2 C \cos 2(P-C)] \sin ^{2} A \\
& +[\sin 2 C \cos 2(P-C) \cos \Delta-\sin 2(P-C) \sin \Delta] \sin 2 A \tan \psi\}
\end{aligned}
$$

where, $K$ is a compound coefficient determined by the light source and the detector and is set as 1 for convenience; $R_{S}$ is the complex reflection coefficients for $\mathrm{p}$ - and s-polarized lights, which could be 


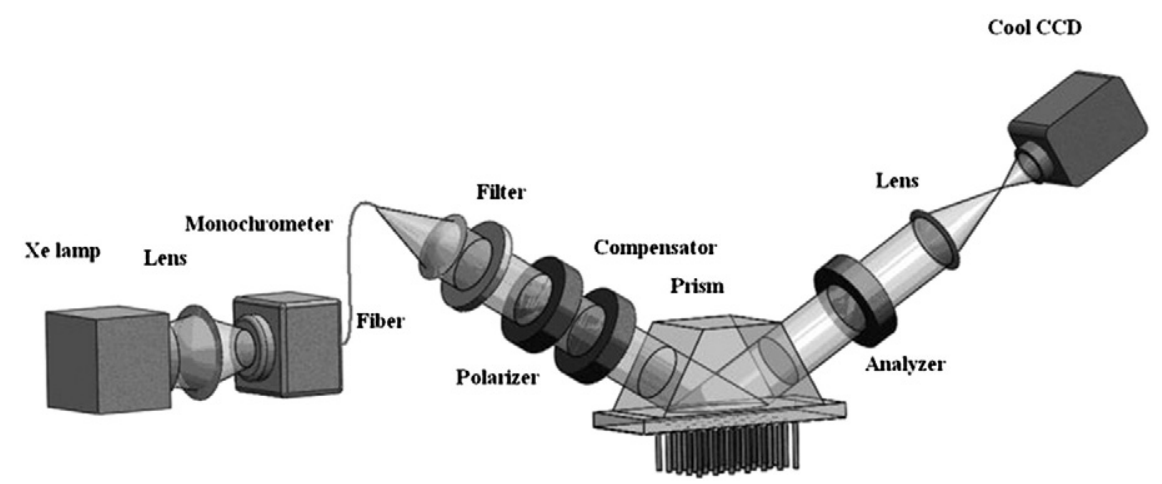

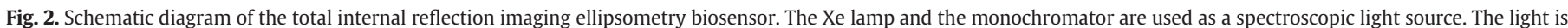

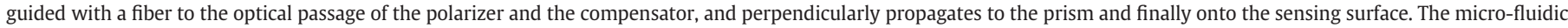

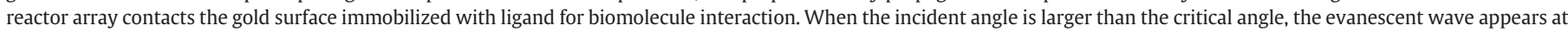
the sensing surface to detect biomolecule interaction. The reflection light passes the analyzer to be imaged by the CCD camera.

deduced from Fresnel equations [11]. $\Psi, \Delta$ and $R_{S}$ are all constants in the sample system with fixed optical parameters. Here, only $P$ and $A$ are considered to vary, since any value of $P-C$ can be obtained by varying $P$ at a fixed $C$, which is assumed to be $45^{\circ}$ without loss of generality. The intensity contrast is a function of the azimuth setting of $P$ and $A$ for a protein layer with a thickness $d_{p r}$ formed by a target of some concentration deduced from Eq. (1), which can be expressed as:

$D=\frac{\Delta I}{I_{i}}=\frac{I\left(P, A, d_{p r}\right)-I_{0}(P, A, 0)}{I_{i}}$.

For the polarization optimization in a practical case, a $3.5 \mathrm{~nm}$ thick immunoglobulin G (IgG) adsorption layer is detected at different settings of the polarizer and the analyzer. The wavelength is set at $633 \mathrm{~nm}$ and the incident angle is at $59^{\circ}$, which are determined by a theoretical optimization. The sample can be simplified as a system of glass/gold layer/protein layer/liquid. The substrate coupled with the prism made of SF10 has the refractive index of 1.72. The thickness and the refractive index of the gold layer are about $30 \mathrm{~nm}$ and $0.14+\mathrm{j} 3.33$, respectively, which are measured with a conventional ellipsometer (WVASE32 J.A. Woollam Co.). The liquid background is the PBS buffer with the refractive index of 1.33 . The azimuth of the polarizer and the analyzer can be adjusted in the range from $0^{\circ}$ to $180^{\circ}$.

The light input is a constant at a certain wavelength such as $633 \mathrm{~nm}$. The normalized intensity contrast response curves against the azimuth of the polarizer and the analyzer from $0^{\circ}$ to $180^{\circ}$ are obtained and shown by a contour map in Fig. 3. The absolute value of the intensity contrast could be obtained from 0 to the maximum. The plot indicates that the optimal settings are $P=110^{\circ}, A=145^{\circ}$ and $P=20^{\circ}, A=35^{\circ}$ corresponding to the highest intensity contrast when the wavelength is $633 \mathrm{~nm}$. It is shown that the maximum intensity contrast could be obtained by polarization optimization at a certain wavelength.

\subsection{Setting of the wavelength}

Apart from the effect of polarization on the sensitivity at a fixed wavelength discussed above, we also have to consider the effect of the wavelength on the sensitivity. The components of the sample system composed of the glass, the gold layer, the protein layer and the liquid all have the property of dispersion, that is, the refractive indexes depend on the wavelength. According to the Fresnel equations, the complex reflection coefficients $R_{S}$ is the functions of the wavelength $\lambda$ when the incident angle and the thickness of the gold layer are fixed. From Eq. (1), the intensity contrast of a protein layer can be determined from the wavelength, the azimuth of the polarizer and the analyzer, as

$$
\frac{\Delta I}{I_{i}}=\frac{I\left(\lambda, P, A, d_{p r}\right)-I_{0}(\lambda, P, A, 0)}{I_{i}} .
$$

In order to optimize wavelength choice, a $3.5 \mathrm{~nm}$ thick IgG protein layer is detected as an example at each wavelength with an interval of $50 \mathrm{~nm}$ within the wavelength range of 450 to $700 \mathrm{~nm}$ by the polarization optimization. The intensity of the input light $I_{i}$ and the intensity difference $\Delta I$ of the protein layer and the substrate are measured. The normalized intensity contrasts are obtained by optimizing the setting of the polarizer and the analyzer at each wavelength. The maximum intensity contrast is obtained when the wavelength is $600 \mathrm{~nm}$, the minimum intensity contrast of the measured wavelength is $450 \mathrm{~nm}$, and the maximum is about 7 times larger than the minimum. The optimal setting of the wavelength and the azimuth of the polarizer and the analyzer is $600 \mathrm{~nm}, 122^{\circ}$ and $44^{\circ}$, respectively, as determined from practical detection.

The above sample system is tested. The phase difference $\delta \Delta$ between IgG protein adsorption layer with a thickness of $3.5 \mathrm{~nm}$ and the liquid background is obtained when the wavelength varies from 450 to $700 \mathrm{~nm}$ which is as shown in Fig. 4. It is shown that $\delta \Delta$ takes the maximum value when the wavelength is $600 \mathrm{~nm}$. In the ellipsometric

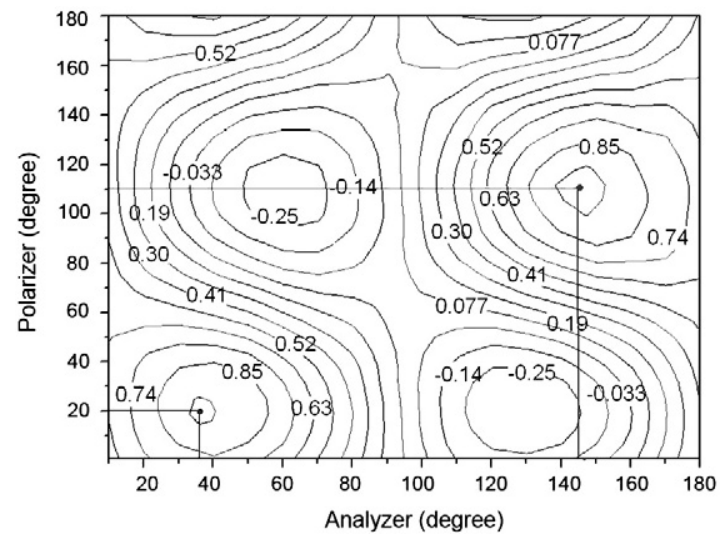

Fig. 3. The normalized intensity difference between the protein layer and the substrate vs. the azimuths of the polarizer and the analyzer. The wavelength is $633 \mathrm{~nm}$, and the angle of incidence is $59^{\circ}$. The glass and the prism made of SF10 have the refractive index of 1.72. The thickness and the refractive index of the gold layer are about $30 \mathrm{~nm}$ and $0.14+\mathrm{j} 3.33$, respectively. The substrate is the PBS buffer with the refractive index of 1.33. The thickness of the protein layer is $3.5 \mathrm{~nm}$. 


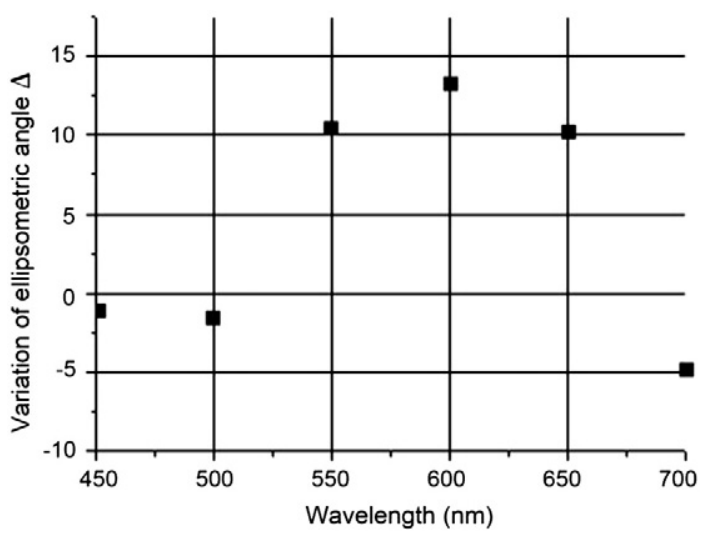

Fig. 4. Phase difference between a $3.5 \mathrm{~nm}$ thick protein layer and the substrate.

measurement, $\Delta$ is more sensitive than $\Psi$ in this experiment and the bigger the phase difference is, the more sensitive the biosensor will be. The white light source of the Xe lamp with a scanning-monochromator has the wavelength with a width around $10 \mathrm{~nm}$ within the spectral range of 450 to $700 \mathrm{~nm}$.

\subsection{Imaging device}

The sensitivity and the detection limit can be improved by increasing the signal to noise ratio. Generally, in the TIRIE biosensor, the noise mainly comes from temperature fluctuation in the imaging device. A general approach to reduce the thermally induced noise is to implement a temperature controller [18] in the imaging device.

In the previous TIRIE biosensor system, the imaging device is a video CCD (Sony XC-30CE) without the temperature control. The sensitivity and the detection limit of the biosensor are not quite satisfactory and can hardly satisfy the demands of biological detection. In the new system, a cool CCD (Andor DU937N-BV) with low noise function is used as the imaging device of the TIRIE biosensor. With low read noise and a temperature control, the cool CCD reduces the thermal noise effectively. In order to illustrate the function of the cool CCD in the biosensor measurement, a $3.5 \mathrm{~nm}$ thick IgG adsorption protein layer is imaged by the low noise CCD and the video CCD. The exposure time of both cameras is $0.04 \mathrm{~s}$ and 100 serial pictures are captured for average shown in Fig. 5. Here, the mean square root is taken to measure the noise of the biosensor. The noise of the biosensor without and with cool CCD is $5 \times 10^{-3}$ and $4.5 \times 10^{-4}$, respectively. It is concluded that the cool CCD with low noise (Andor DU937N-BV, read noise $10.3 \mathrm{e}$ at $2.5 \mathrm{MHz}$ dark current $0.0005 \mathrm{e}-/ \mathrm{pixel} / \mathrm{s}$ at $-80^{\circ} \mathrm{C}$ ) as the imaging device can improve SNR by more than 10 times.

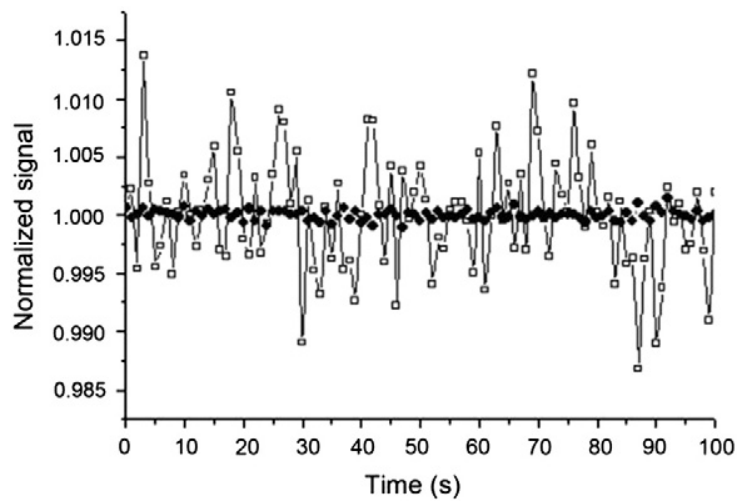

Fig. 5. The normalized signals of the $3.5 \mathrm{~nm}$ thick $\operatorname{IgG}$ protein layer obtained by the biosensor with video CCD (“ $\square$ ”) and cool CCD (“•”).

\section{Experiments}

The purpose of the optimization is to find out the optimal setting for the analyte to be detected with high sensitivity and wide detection limit. An experiment is performed to verify the performance improvement of the biosensor. It concerns the quantitative detection of Hepatitis B surface antigen (HBsAg). The optical conditions of the biosensor are as described above.

\subsection{Materials}

The SF10 glass slides were provided by the Changchun Institute of Optics, Fine Mechanics and Physics, Chinese Academy of Sciences (China), the 11-mercaptoundecanoic acid (MUA) was from Sigma (USA), 1-(3-dimethylaminopropyl)-3-ethylcarbodimide hydrochloride (EDC) and N-hydroxy-droxysuccinimide (NHS) were from ACROS, and all chemicals for preparing phosphate-buffered saline (PBS, $10 \mathrm{mM}$ phosphate, $0.1 \mathrm{M} \mathrm{NaCl}, \mathrm{pH}$ 7.4) were from Sigma also. De-ionized water (with a resistivity of $18.3 \mathrm{M} \Omega \mathrm{cm}$ ) was produced by ion exchange demineralization, followed by passing through a Milli-Q plus system from Millipore (Millipore, Bedford, MA). Hepatitis B virus surface antibody and surface antigen were provided by the Hotgen company (Beijing China) and the blocking buffer was from Sigma (USA).

\subsection{Substrate}

The SF10 glass slide was prepared by the evaporation of $2 \mathrm{~nm}$ chromium on the surface and $30 \mathrm{~nm}$ gold layer was used as the substrate. The gold surface was immersed into MUA-ethanol solution for at least $18 \mathrm{~h}$, followed by a thorough rinsing with both ethanol and de-ionized water. The MUA monolayer with a carboxyl group was self-assembled on the gold surface.

\subsection{Experimental setup}

The incident angle is $59^{\circ}$. The wavelength is $600 \mathrm{~nm}$. The azimuths of the polarizer and the analyzer are $122^{\circ}$ and $44^{\circ}$, respectively, according to the optimization setting. The temperature of the CCD during the detection is $-70^{\circ}$. The pre-gain of the CCD is 2 and the dynamic range in grayscale is from 0 to 65,535 .

\section{Results}

The gold surface with a carboxyl group on is activated with NHS and EDC then rinsed with PBS buffer. The Hepatitis B surface antibody (HBsAb) is used as the ligand and covalently immobilized on the gold surface with a carboxyl group for $10 \mathrm{~min}$. The surface is rinsed for about 6 min to remove unbound molecules. Then it is blocked with blocking buffer for $10 \mathrm{~min}$, and rinsed with PBS buffer for $6 \mathrm{~min}$. The HBsAg is diluted with PBS buffer into various concentrations from 0 to $250 \mathrm{ng} / \mathrm{ml}$ and incubated with ligand for interactions upon their affinity for $15 \mathrm{~min}$. The whole dynamic process of the interaction between HBsAb and HbsAg is shown in Fig. 7, where it shows an average result over each sensing area in each cell in the array of 100 pixels. The curves in Fig. 6 are zoomed into "region 7" and "region 8 " to show the dynamic process of the interaction in detail on the surface. Curves from "a" to "g" correspond to HbsAg concentrations of $0,8,16,32,63,125$, and $250 \mathrm{ng} / \mathrm{ml}$. The data in "region 8 " is averaged over the last $600 \mathrm{~s}$, as indicated in Fig. 6.

\section{Discussions}

The results of the experiment show the improvement of the sensitivity and the detection limit of the TIRIE biosensor with the techniques proposed in this paper. 


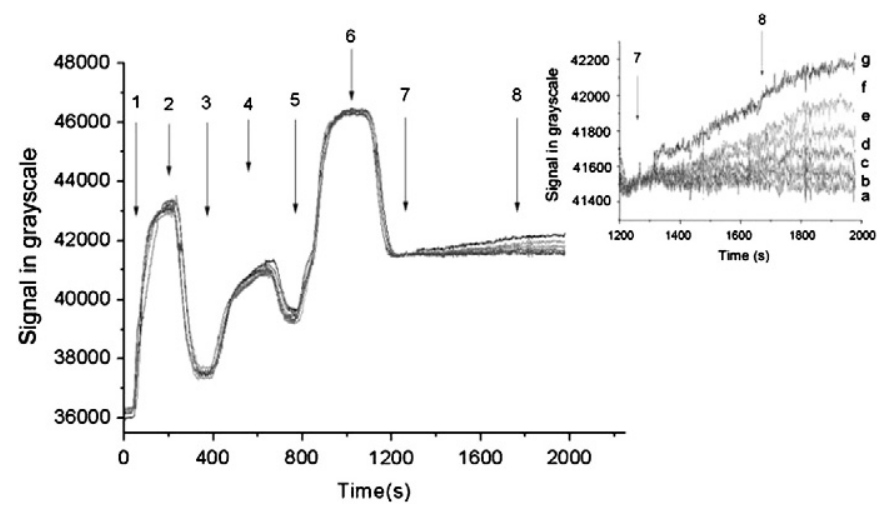

Fig. 6. Real-time curves of the dynamic process for the Hepatitis B virus surface antigen binding with Hepatitis B surface antibody in various concentrations. The 1st region is the baseline; the 2nd region - NHS-EDC activation of the carboxyl group assembled on the gold substrate; 3rd - PBS rising; 4th - HBsAb immobilization on the gold-coated substrate surface; 5 th - PBS rinsing, 6 th - blocking , 7th - PBS rinsing and 8th - HBsAg binding with its antibody. Curves $\mathrm{a}-\mathrm{h}$ are for Hepatitis $\mathrm{B}$ virus surface antigen concentrations of $0,8,16,32,63,125$, and $250 \mathrm{ng} / \mathrm{ml}$.

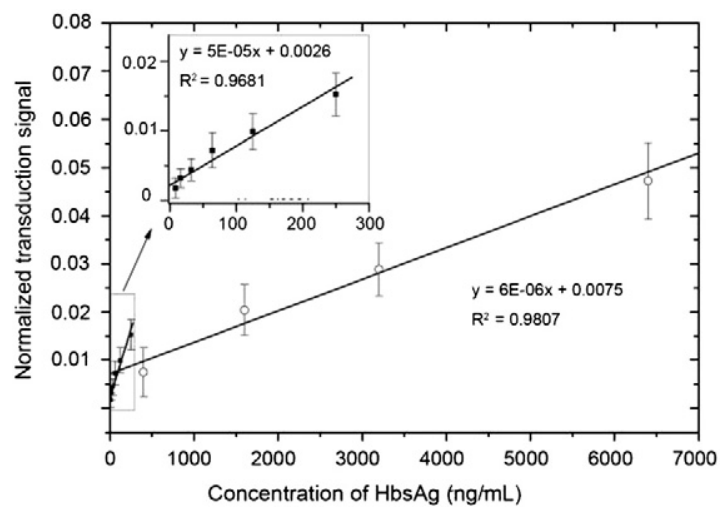

Fig. 7. The normalized transduction signal versus the concentration of $\mathrm{HbsAg}$. The zoomed curve represents the data obtained by the biosensor after optimization.

The normalized transduction signal versus the concentrations of HbsAg obtained from the previous biosensor system and the improved system is shown in Fig. 7. The slope of the regressive curve is $6 \times 10^{-6}$ and $5 \times 10^{-5}$, respectively, which are taken as the sensitivity of the biosensor system. Obviously, the sensitivity is improved by almost one order.

Fig. 8 shows kinetic curves of HBsAb reacting with various concentrations of HBsAg for the previous system and the improved system. The transduction signals are normalized by the mean value of the initial $100 \mathrm{~s}$ of the dynamic curves, as the baseline of the interaction. Curves "D" to "A" with concentrations 400, 1600, 3200, $6400 \mathrm{ng} / \mathrm{ml}$ are obtained by the previous biosensor system. The wavelength is set as $633 \mathrm{~nm}$ and the imaging device is the video CCD camera (Sony XC-ST30 CCD B/W video camera). Curves "k" to "e" with concentrations of $0,8,16,32,63,125,250 \mathrm{ng} / \mathrm{ml}$ are obtained by the improved biosensor with optimized conditions mentioned above.

The detection limit of the previous biosensor is $400 \mathrm{ng} / \mathrm{ml}$, as being affected by the noise. The signal can hardly be distinguished when the concentration is lower. The performance of the detection limit is improved from $400 \mathrm{ng} / \mathrm{ml}$ to $8 \mathrm{ng} / \mathrm{ml}$ by optimizing the setting of the wavelength, and the azimuth setting of the polarizer and the analyzer as well as by using the cool CCD. The detection limit in the improved biosensor system is enhanced by about 50 times.

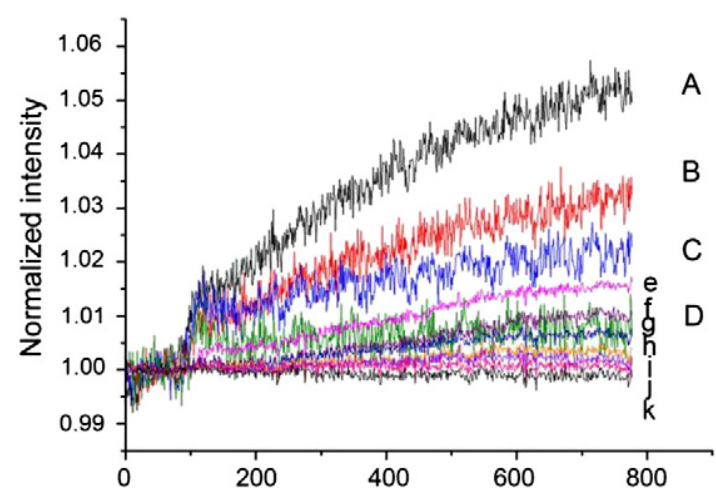

Fig. 8. Dynamic curves of HbsAg reacting with HbsAb: curves "D" to "A" represent the normalized signal with concentrations of 400 to $6400 \mathrm{ng} / \mathrm{ml}$ measured without optimized conditions; curves " $\mathrm{k}$ " to " $\mathrm{e}$ " represent the normalized signal with concentrations of 0 to $250 \mathrm{ng} / \mathrm{ml}$ measured under optimized conditions.

\section{Conclusion}

In this paper, we show that the sensitivity and the detection limit are improved by the setting optimization of polarization components and wavelength as well as by using a cool CCD. The sensitivity is increased by about one order and the detection limit is increased 50 times, as demonstrated by the quantitative detection of the HbsAg.

The potential application in detection of biomolecule weak-affinity interaction and clinic test with high throughput can be foreseen.

\section{Acknowledgements}

The author gratefully acknowledges the support of the National Basic Research Program of China (2009CB320300). The National High Technology Research and Development Program (863) of China (2008AA02Z419), the Chinese Academy of Sciences (KJCX2-YW-M03 and KJCX2-YW-M04), and the National Natural Science Foundation of China 20805053.

\section{References}

[1] R. Narayanaswamy, O.S. Wolfbeis, Optical Biosensors, Springer, New York, 2004

[2] A. Rasooly, J. Jacobson, Biosens. Bioelectron. 21 (2006) 1851.

[3] F. Ricci, G. Volpe, L. Micheli, G. Palleschi, Anal. Chim. Acta 605 (2007) 111.

[4] S. Lofas, M. Malmqvist, I. Ronnberg, E. Stenberg, B. Liedberg, I. Lundstrom, Sens. Actuators, B 5 (1991) 79.

[5] M. Malmqvist, Nature 361 (1993) 186

[6] E. Kretschm, Z. Phys. 241 (1971) 313.

[7] A.V. Kabashin, V.E. Kochergin, P.I. Nikitin, Sens. Actuators, B 54 (1999) 51.

[8] K. Nagata, H. Handa, Real-time Analysis of Biomolecular Interactions, Springer-Verlag, Tokyo, 2000.

[9] R.L. Rich, A.R. Miles, B.K. Gale, D.G. Myszka, Anal. Biochem. 386 (2009) 98

[10] M.A. Eddings, A.R. Miles, J.W. Eckman, J. Kim, R.L. Rich, B.K. Gale, D.G. Myszka, Anal. Biochem. 382 (2008) 55.

[11] R.M.A. Azzam, N.M. Bashara, Ellipsometry and Polarized Light, 1977, North-Holland, New York.

[12] H. Arwin, M. Poksinski, K. Johansen, Appl. Opt. 43 (2004) 3028.

[13] A.V. Nabok, A. Tsargorodskaya, A.K. Hassan, N.F. Starodub, Appl. Surf. Sci. 246 (2005) 381.

[14] M.J. Jory, G.W. Bradberry, P.S. Cann, J.R. Sambles, Meas. Sci. Technol. 6 (1995) 1193.

[15] P. Westphal, A. Bornmann, Sens. Actuators, B 84 (2002) 278.

[16] W. Yuan, H.P. Ho, S.Y. Wu, Y.K. Suen, S.K. Kong, Sens. Actuators, A 151 (2009) 23.

[17] Y.Y. Chen, J. Gang, Biosens. Bioelectron. 219 (2006).

[18] X. Fan, I.M. White, S.I. Shopova, H. Zhu, J.D. Suter, Y. Sun, Anal. Chim. Acta 620 (2008) 8.

[19] I.M. White, X.D. Fan, Opt. Express 16 (2008) 1020. 